\title{
A 2003 stratospheric aerosol extinction and PSC climatology from GOMOS measurements on Envisat
}

\author{
F. Vanhellemont ${ }^{1}$, D. Fussen ${ }^{1}$, C. Bingen ${ }^{1}$, E. Kyrölä ${ }^{2}$, J. Tamminen ${ }^{2}$, V. Sofieva ${ }^{2}$, S. Hassinen ${ }^{2}$, P. Verronen ${ }^{2}$, \\ A. Seppälä ${ }^{2}$, J. L. Bertaux ${ }^{3}$, A. Hauchecorne ${ }^{3}$, F. Dalaudier ${ }^{3}$, O. Fanton d'Andon ${ }^{4}$, G. Barrot ${ }^{4}$, A. Mangin ${ }^{4}$,

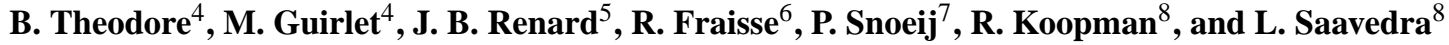 \\ ${ }^{1}$ Belgian Institute for Space Aeronomy, Brussels, Belgium \\ ${ }^{2}$ Finnish Meteorological Institute, Earth Observation, Helsinki, Finland \\ ${ }^{3}$ Service d'Aéronomie du CNRS, Verrieres-le-Buisson cedex France \\ ${ }^{4}$ ACRI-ST, Sophia-Antipolis, France \\ ${ }^{5}$ CNRS-LPCE, Orléans, France \\ ${ }^{6}$ Astrium SAS, Toulouse cedex France \\ ${ }^{7}$ ESTEC (ESA), Noordwijk, The Netherlands \\ ${ }^{8}$ ESRIN (ESA), Frascati, Italy
}

Received: 25 November 2004 - Published in Atmos. Chem. Phys. Discuss.: 16 February 2005

Revised: 17 August 2005 - Accepted: 5 September 2005 - Published: 20 September 2005

\begin{abstract}
Stratospheric aerosols play an important role in a number of atmospheric issues such as midlatitude ozone depletion, atmospheric dynamics and the Earth radiative budget. Polar stratospheric clouds on the other hand are a crucial factor in the yearly Arctic and Antarctic ozone depletion. It is therefore important to quantify the stratospheric aerosol/PSC abundance. In orbit since March 2002, the GOMOS instrument onboard the European Envisat satellite has provided a vast aerosol extinction data set. In this paper we present aerosol/PSC zonal median values that were constructed from this data set, together with a discussion of the results.
\end{abstract}

\section{Introduction}

Since its discovery (Junge et al., 1961), the stratospheric aerosol layer has gained increasing attention because of its role in a number of atmospheric phenomena. The role of these aerosols at midlatitudes in heterogeneous ozone chemistry was discovered only recently (Solomon et al., 1996). Due to their optical scattering and absorption properties, aerosols have a significant impact on the Earth radiative budget (Dutton and Christy, 1992), and hence on the global climate. Stratospheric aerosols primarily consist of droplets of a sulfuric acid solution, of which the source gas $\mathrm{SO}_{2}$ is injected in the stratosphere by strong volcanic eruptions. Events like these can increase the stratospheric aerosol loading by several orders of magnitude. The last volcanic erup-

Correspondence to: F. Vanhellemont

(filip.vanhellemont@oma.be) tion of this strength, Mount Pinatubo in the Philippines, occured in 1991. It has provided an excellent opportunity to study the impact of aerosols on ozone chemistry, climate and atmospheric dynamics (for a general review on volcanism and the atmosphere, see Robock, 2000). Since 1991, due to slow sedimentation, the aerosol abundance has gradually decreased to the present day level, the lowest since decades. Nevertheless, the absorption/scattering efficiency of aerosols is such that they still show their imprint in a wide range of optical measurements.

Polar Stratospheric Clouds (PSCs) are usually discussed separately from sulfuric acid aerosols because the particles of which they consist have a different state, morphology and composition and because they only form at extremely low temperatures in the polar stratosphere. PSCs receive a lot of attention because Arctic and Antarctic ozone depletion is mainly induced by heterogeneous reactions on the surface of PSC particles (Solomon et al., 1986; Molina, 1991).

In this paper we present stratospheric aerosol/PSC zonal median values for the year 2003 that were derived from measurements carried out by the GOMOS instrument onboard the European Envisat satellite. Such values serve many purposes. In a qualitative way, a global picture of aerosols and PSCs gives insight into the reasons for the variability of these species. Furthermore, zonal median values can be used in other studies that need a quantitative characterization of aerosols and PSCs, such as stratospheric chemistry modelling, optical calculations and measurement corrections. We present results for the entire year, as well as for 3-month periods to show the interseasonal variability.

(C) 2005 Author(s). This work is licensed under a Creative Commons License. 


\section{The GOMOS instrument}

The Envisat satellite was launched on 1 March 2002, and is at present fully operational. On board, it carries a range of instruments designed to measure data with specific application in a wide diversity of Earth science studies. One of these instruments, GOMOS (Global Ozone Monitoring by Occultation of Stars; see e.g. Bertaux et al., 1991, 2000; Kyrölä et al., 2004; ESA, 2001) is a UV/Visible/near-IR spectrometer that works in occultation mode: while orbiting the Earth, the instrument measures the transmission of light from stars that are setting below the Earth's horizon. Since the starlight has to pass through the Earth's atmosphere, it is partly scattered or absorbed by atmospheric gases and particles. The measurements therefore can be used to retrieve gas concentration and aerosol extinction profiles. Using a scanning mirror and a star tracker, GOMOS continuously observes selected stars; during one orbit, several different occultations are measured. In this way, several hundreds of occultations with good global coverage can be measured per day. Measurements are taken both on the dark and Sun-illuminated side of the Earth, although in the latter case scattered sunlight represents an extra source of error for retrievals. Given the fact that during one orbit about 30 to 50 ocultations can be measured, the entire data set for the year 2003 contains more than 100000 occultations.

The spectrum of the starlight is measured by four spectrometers operating in a wavelength range from 250 to $950 \mathrm{~nm}$. Additionally, GOMOS is equipped with two fast photometers of which the signals are used to correct for star scintillation and to retrieve high-resolution temperature profiles. While GOMOS originally was conceived as an instrument designed to measure highly accurate ozone profiles, a few other species can also be derived. Typically, the UV/Vis wavelength range combined with the sensitivity of the GOMOS spectrometers allows the retrieval of ozone, $\mathrm{NO}_{2}$, air, $\mathrm{NO}_{3}, \mathrm{O}_{2}$ and aerosol extinction profiles. While ozone can be retrieved up to $100 \mathrm{~km}$ of altitude, the other species are usually only detectable from the upper troposphere to about $50 \mathrm{~km}$.

\section{GOMOS retrievals}

The current GOMOS algorithm works in two steps (for more details, see Kyrölä et al., 1993; ESA, 2001). First, all measured transmittance spectra are inverted to slant path integrated density (for gases) and optical thickness (for aerosols). This spectral inversion step is performed for all tangent altitudes separately, using a nonlinear least-squares algorithm. Second, using the matrix of weighting functions, these data are spatially inverted to local gas concentration and aerosol extinction profiles. Since the measurements are contaminated with residual star scintillation due to the imperfect demodulation by the fast photometers, this spatial inversion is performed with a Tikhonov regularization method, where the regularization parameter is chosen such that some predetermined target resolution is achieved.

Unlike the case for gases, where the extinction cross sections are known from laboratory measurements, the aerosol extinction spectrum is a priori unknown. Typically, some parametrized function of wavelength is used (such as a polynomial, see e.g. Fussen et al., 2001; Berthet et al., 2002), with sufficient degrees of freedom to capture the aerosol extinction behaviour. However, the random error variance of the other constituent retrievals increases with the number of aerosol parameters. This is the case for ozone, still the main target constituent of the GOMOS mission. It is certainly the case for air retrievals, since the air and aerosol extinction spectra are both very smooth functions of wavelength. To avoid this problem, it was decided to describe the aerosol extinction with one parameter:

$\beta_{a}(\lambda)=N \frac{\sigma_{0}}{\lambda}$

with the scaling factor $\sigma_{0}=3.10^{-7} \mathrm{~cm}^{2} \mathrm{~nm}$ and $N$ (unit: $\mathrm{cm}^{-3}$ ) the parameter to be retrieved.

The reason for this analytical form is twofold. First, it is a decreasing function of wavelength, roughly corresponding to the extinction spectrum of moderately small particles, such as the ones that populate the 2003 stratospheric aerosol layer. And second, the decrease is less steep than the $1 / \lambda^{4}$ Rayleigh limit, thus reducing the interdependence of aerosol and air retrievals.

Some criticism is appropriate here. While a $1 / \lambda$ model may be able to describe typical background aerosol spectra, it will fail to represent large aerosol particles in volcanic periods, for which is known that they exhibit flatter spectra or show maxima in the visible wavelength range. On the other size range, for extremely small particles, the physical aerosol spectrum is by definition indistinguishable from the $1 / \lambda^{4}$ air spectrum. In between these extreme cases, we find an entire gamut of possible spectra, some of which will look very different from our model. For all these cases, the retrievals with our model will probably be biased with respect to the actual situation. Furthermore, we should mention that Eq. (1) represents an aerosol model of which the form of the extinction spectrum is constant in altitude, a rather limited description of reality.

\section{Data processing}

As mentioned before, GOMOS is able to take measurements at the Sun-illuminated as well as the dark side of the Earth. For day side observations, the measurements are corrected by subtracting the bright limb component, that is measured with the upper and lower bands of the CCD detector. Nevertheless, after subtraction, the residual noise is large enough to significantly decrease the retrieval accuracy. We therefore 

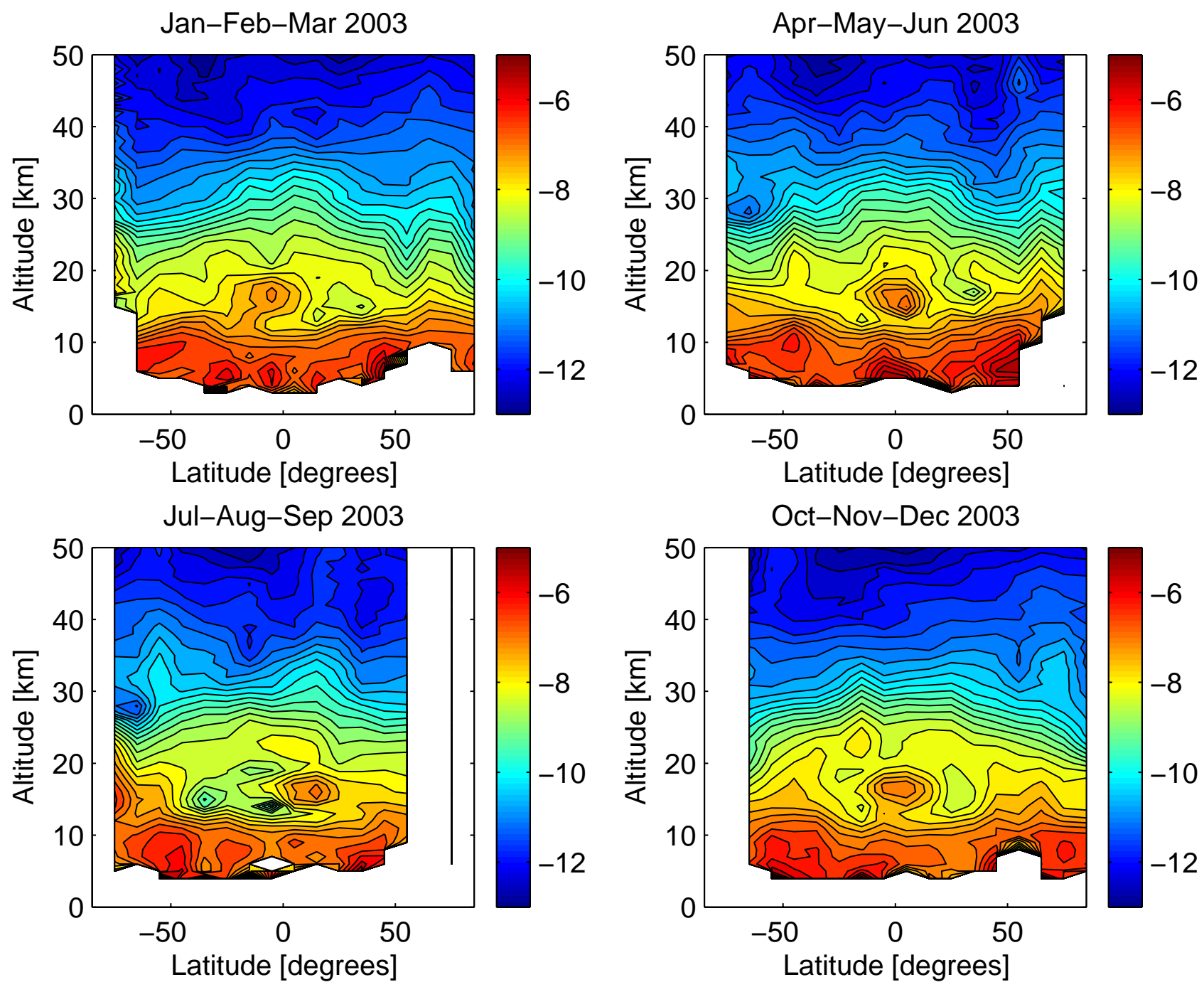

Fig. 1. The natural logarithm of the $500 \mathrm{~nm}$ aerosol extinction median values for 4 different periods of the year 2003. From top left to bottom right: January/February/March, April/May/June, July/August/September, October/November/December 2003. Notice the movement of the maximum in the tropics, and the appearance of Polar Stratospheric Clouds in the Antarctic lower stratosphere during July/August/September.

decided to use only night-time measurements. After this data selection, about 50000 occultations were left to use.

The spatial grid that we use consists of 18 latitude bins with a width of 10 degrees, with bin centers ranging from $85^{\circ} \mathrm{S}$ to $85^{\circ} \mathrm{N}$. The altitude grid consists of bins having a width of $1 \mathrm{~km}$, and centers ranging from 0 to $60 \mathrm{~km}$. No variations along longitude are considered. We furthermore use monthly bins, from January until December 2003.

Every bin contains a number of data points, of which the statistical distribution is a priori unknown. From visual inspection, we found that many distributions were asymmetric, with occasional strong outliers. In such cases, the statistical mean is a poor way to describe the central tendency of the distribution, and therefore the use of the median (or 0.5 percentile) was our preferred estimator. In addition, the $0.1,0.2$, $0.3,0.4,0.6,0.7,0.8$ and 0.9 percentiles were calculated to describe the variance of the distributions. Typically, there are a few hundred data points in each bin.

\section{Results}

Figure 1 shows four plots of the logarithm of aerosol extinction at $500 \mathrm{~nm}$, calculated for 3-month periods: January to March, April to June, July to September and October to December. Clearly visible is the umbrella-shaped aerosol layer, having the largest altitude above the equator and gradually sloping downwards when moving to the poles. An interesting feature is the isolated maximum in the tropics at an altitude of 15 to $17 \mathrm{~km}$, that gradually moves towards the North, and then heads back southwards at the end of the year. The maximum has been observed by other instruments as well 

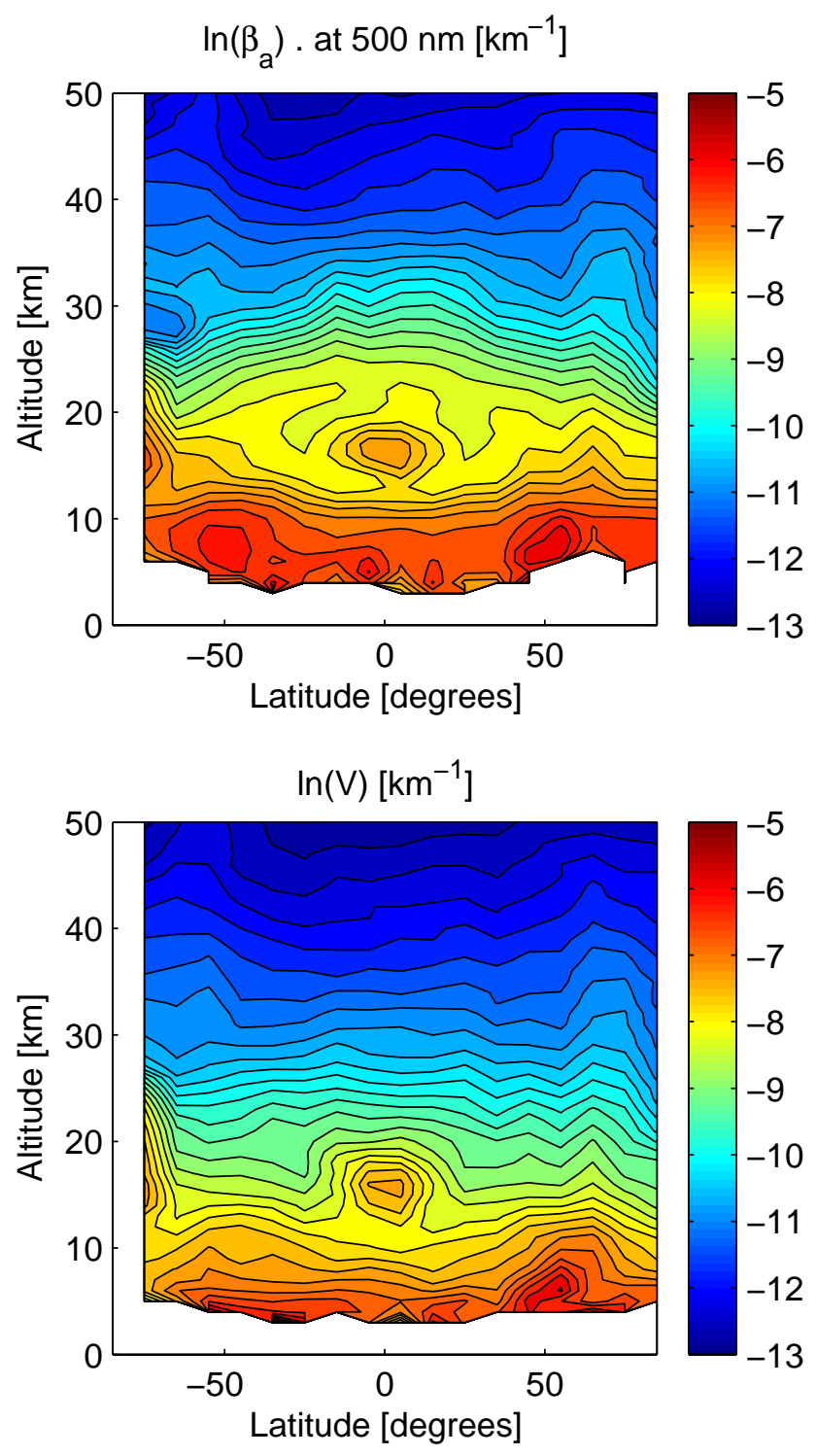

Fig. 2. Top: the natural logarithm of the $500 \mathrm{~nm}$ aerosol extinction median values for the year 2003. Bottom: the $500 \mathrm{~nm}$ aerosol extinction variability as defined in Eq. (2).

and is caused by the presence of high subvisual cirrus clouds in the tropics, while the movement is in phase with the seasonal shift of the Intertropical Convergence Zone (ITCZ) (see Wang et al., 1996). Also interesting are the elevated levels in the Antarctic region in the period July to September, most certainly caused by the occurence of PSCs in the Antarctic vortex during winter. However, no elevated extinction levels are observed in the Arctic region from January to March. This is explained by the much lower occurence of PSCs, due to the weaker polar vortex.

The $500 \mathrm{~nm}$ aerosol extinction zonal median values for the entire year 2003 are presented in Fig. 2. The first plot again shows the logarithm of the $500 \mathrm{~nm}$ aerosol extinction, while

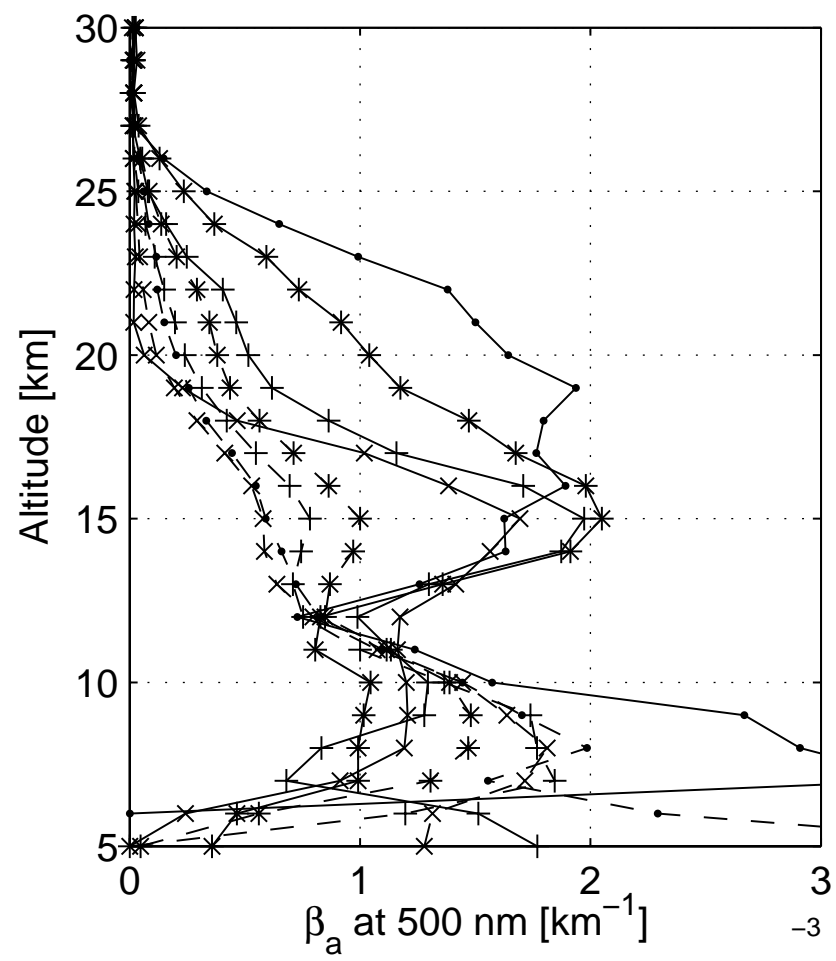

Fig. 3. The $500 \mathrm{~nm}$ aerosol extinction median values for June (dots), July (asterisks), August (+) and September (x) 2003, inside $\left(75^{\circ} \mathrm{S}\right.$; solid lines) and outside $\left(65^{\circ} \mathrm{S}\right.$; dashed lines) the South polar vortex. Data for the $85^{\circ} \mathrm{S}$ bin were not available for these months. The PSC signature is clearly present in the lower stratosphere above $12 \mathrm{~km}$.

the second plot shows a representation of the yearly variance, calculated with percentiles:

$V=\frac{p_{0.8}-p_{0.2}}{2}$

This plot shows most clearly where the strongest variations in aerosol extinction are present: the already mentioned maximum in the tropics, and the Antarctic appearing and disappearing of PSCs (giving rise to strong variations up to altitudes as high as $28 \mathrm{~km}$ ).

In Fig. 3, the PSC phenomenon is presented with more detail. Shown are median values for the months June, July, August and September, for latitude bins $85^{\circ} \mathrm{S}$ and $75^{\circ} \mathrm{S}$, roughly corresponding to locations respectively inside and outside the polar vortex. While the profiles outside the vortex maintain a more or less constant shape during the entire period, one can observe a strong enhancement in the lower stratosphere inside the vortex. Furthermore, the peak gradually descends to lower altitudes as time passes by, an observation that is likely caused by the combination of downward transport, sedimentation and changing temperature. 


\section{Conclusions}

The presented zonal median values were constructed from the night-time subset of the entire 2003 GOMOS data set. Although recent aerosol values are extremely low, GOMOS has been able to provide quality aerosol extinction profiles, that exhibit all the features that ought to be expected. The typical shape of the aerosol layer, and the occurence of PSCs within the Antarctic winter vortex are only the two most prominent features that are observed in the data set. In the near future, when the quality of the bright limb retrievals will be much better, much more profiles will be available to improve the data set. Furthermore, an additional study will be performed to find a more adequate model for the spectral extinction behaviour of aerosols and PSCs. The general conclusion from the results presented here is that GOMOS will be able to provide a quality long-term aerosol climatology that can be used in a wide field of studies.

Acknowledgements. This work was financially supported with the Prodex 7 contract "SADE" (MO/35/009), granted by the Federal Office for Scientific, Technical and Cultural Affairs (OSTC) of the Belgian government.

Edited by: A. Lambert

\section{References}

Bertaux, J. L., Megie, G., Widemann, J., Chassefiere, E., Pellinen, R., Kyrölä, E., Korpela, S., and Simon, P.: Monitoring of ozone trend by stellar occultations: The GOMOS instrument, Adv. Space Res., 11, 237-242, 1991.

Bertaux, J. L., Kyrölä, E., and Wehr, T.: Stellar occultation technique for atmospheric ozone monitoring: GOMOS on Envisat, Earth Observation Quarterly, 67, 17-20, 2000.

Berthet, G., Renard, J.-B., Brogniez, C., Robert, C., Chartier, M., and Pirre, M.: Optical and physical properties of stratospheric aerosols from balloon measurements in the visible and nearinfrared domains. I. Analysis of aerosol extinction spectra from the AMON and SALOMON balloonborne spectrometers, Appl. Opt., 41, 7522-7539, 2002.
Dutton, E. and Christy, J.: Solar radiative forcing at selected locations and evidence for global lower tropospheric cooling following the eruptions of El Chichón and Pinatubo, Geophys. Res. Lett., 19, 2313-2316, 1992.

ESA: Envisat-GOMOS - An instrument for global atmosphere ozone monitoring, Technical Report SP-1244, European Space Agency, 2001.

Fussen, D., Vanhellemont, F., and Bingen, C.: Remote Sensing of the Earth's atmosphere by the spaceborne Occultation Radiometer, ORA: final inversion algorithm, Appl. Opt., 40, 941-948, 2001.

Junge, C., Chagnon, C., and Manson, J.: Stratospheric aerosols, J. Meteor., 18, 80-108, 1961.

Kyrölä, E., Sihvola, E., Kotivuori, Y., Tikka, M., Tuomi, T., and Haario, H.: Inverse theory for occultation measurements, 1, Spectral inversion, J. Geophys. Res., 98, 7367-7381, 1993.

Kyrölä, E., Tamminen, J., Leppelmeier, G., Sofieva, V., Hassinen, S., Bertaux, J., Hauchecorne, A., Dalaudier, F., Cot, C., Korablev, O., Fanton d' Andon, O., Barrot, G., Mangin, A., Théodore, B., Guirlet, M., Etanchaud, F., Snoeij, P., Koopman, R., Saveedra, L., Fraisse, R., Fussen, D., and Vanhellemont, F.: GOMOS on Envisat: an overview, Adv. Space Res., 33, 10201028, 2004.

Molina, M.: Heterogeneous chemistry on polar stratospheric clouds, Atmos. Environ., 25A, 2535-2537, 1991.

Robock, A.: Volcanic eruptions and climate, Rev. Geophys., 38,2, 191-219, 2000.

Solomon, S., Garcia, R., Rowland, F., and Wuebbles, D.: On the depletion of Antarctic ozone, Nature, 321, 755-758, 1986.

Solomon, S., Portmann, R., Garcia, R., Thomason, L., Poole, L., and McCormick, M.: Role of aerosol variations in anthropogenic ozone depletion at northern midlatitudes, J. Geophys. Res., 101, 6713-6728, 1996.

Wang, P., Minnis, P., McCormick, M., Kent, G., and Skeens, K.: A 6-year climatology of cloud occurrence frequency from Stratospheric Aerosol and Gas Experiment II observations (19851990), J. Geophys. Res., 101, 29407-29429, 1996. 